\title{
Dynamics of colloidal suspensions of ferromagnetic particles in plane Couette flow: Comparison of approximate solutions with Brownian dynamics simulations
}

\author{
Patrick Ilg, ${ }^{1, *}$ Martin Kröger, ${ }^{1,2}$ Siegfried Hess, ${ }^{1}$ and Andrey Yu. Zubarev ${ }^{3}$ \\ ${ }^{1}$ Institut für Theoretische Physik, Technische Universität Berlin, Hardenbergstrasse 36, D-10623 Berlin, Germany \\ ${ }^{2}$ ETH Zürich, Department of Materials, Institute of Polymers, CH-8092 Zürich, Switzerland \\ ${ }^{3}$ Urals State University, Lenin Avenue 51, 620083 Ekaterinburg, Russia
}

(Received 7 February 2003; published 11 June 2003)

\begin{abstract}
The stationary and oscillatory properties of dilute ferromagnetic colloidal suspensions in plane Couette flow are studied. Analytical expressions for the off-equilibrium magnetization and the shear viscosity are obtained within the so-called effective field approximation. We also investigate the predictions of a different approximation based on the linearized moment expansion. Direct numerical simulation of the kinetic model are performed in order to test the range of validity of these approximations.
\end{abstract}

DOI: 10.1103/PhysRevE.67.061401 PACS number(s): 83.80.Hj, 47.65.+a, 75.50.Mm, 05.20.Dd

\section{INTRODUCTION}

The prediction of macroscopic properties of magnetic fluids, such as the magnetization or viscous properties, from a microscopic model remains-despite much efforts - a problem of current research [1-3]. We here obtain approximate expressions of macroscopic properties and test their range of validity by comparison with Brownian dynamics simulations of the underlying kinetic model.

For very dilute systems, a kinetic model has been proposed in Ref. [4] that successfully describes many experimental results. For nondilute ferromagnetic colloidal suspensions a general statistical theory remains to be developed. In order to account for the effects of chain formation in nondilute suspensions, the authors of Refs. $[5,6]$ have proposed a phenomenological extension of the kinetic model for very dilute suspensions, which is able to describe several rheological properties of ferrofluids in agreement with experimental results $[7,8]$. It should be mentioned that different aspects of chain formation are already studied (see, e.g., Ref. [9], and references therein). Similar models are also used to describe magneto- and electrorheological fluids (see, e.g., Ref. [10], and references therein).

In Refs. [6,8], an approximate solution to the extended kinetic model was employed in order to obtain its macroscopic viscous properties. Alternative approximations to this model have been considered in Refs. [3,11]. While the quality of different approximations to the kinetic model of dilute systems, Ref. [4], has been discussed in the literature (see Refs. $[12,13])$, we here compare the predictions of different approximations for the macroscopic magnetization and viscous properties to the numerical solution of the kinetic model of Ref. [6]. No comparison on the level of distribution functions is made since the approximations are constructed to derive macroscopic properties. We consider the case of weak stationary and small amplitude oscillatory Couette flow. For a discussion of a particular approximation to the offequilibrium magnetization in a more general flow situation see Ref. [14]. Numerical results of viscous properties for

*Email address: ilg@physik.tu-berlin.de various shear rates in stationary plane Couette flow with the magnetic field oriented in gradient direction are presented in Ref. [15].

\section{MODEL DEFINITION}

We consider a dilute solution of $n$ identical rigid ellipsoidal ferromagnetic particles per unit volume in a nonpolar Newtonian solvent. The shape of the ellipsoidal particles is completely described by the axis ratio $r$. In Refs. [6,7], a distribution of axis ratios $r$ are considered in order to account for polydispersity effects in the chain-formation process. Since in Refs. [6,7] the chains are assumed to be noninteracting, it is sufficient to consider a monodisperse system in the sequel in order to discuss different approximation to the microscopic dynamics.

We assume that the orientation $\boldsymbol{u}$ of an ellipsoidal particle coincides with the anisotropy axis of the particle. Then, the magnetic moment of a particle is given by $\boldsymbol{\mu}=\boldsymbol{\mu} \boldsymbol{u}$, where $\mu$, the magnitude of the magnetic moment of a particle, is constant. Let $f(\boldsymbol{u} ; t)$ denote the probability distribution function of a ferromagnetic particle being oriented parallel to the unit vector $\boldsymbol{u}$ at time $t$. The normalization is chosen such that $\int d^{2} u f(\boldsymbol{u} ; t)=1$, where the integration is performed over the three-dimensional unit sphere.

The time evolution of $f$ in the presence of a local potential $V(\boldsymbol{u})$ and a velocity field $\boldsymbol{v}(\boldsymbol{r})$ is given by the kinetic equation $[2,6,11]$

$$
\partial_{t} f=-\mathcal{L} \cdot\left\{\left(\boldsymbol{\Omega}+B \boldsymbol{u} \times \boldsymbol{D} \cdot \boldsymbol{u}-\frac{1}{\zeta_{r}}[\mathcal{L} V]\right) f\right\}+D_{r} \mathcal{L}^{2} f
$$

In Eq. (1), we have introduced the rotational operator $\mathcal{L}=\boldsymbol{u} \times \partial / \partial \boldsymbol{u}$, the friction coefficient $\zeta_{r}$ of an ellipsoidal particle with axis ratio $r$ in a Newtonian solvent with viscosity $\eta_{\mathrm{s}}$. The diffusion coefficient $D_{r}=k_{B} T / \zeta_{r}$, with $k_{B}, T$ Boltzmann's constant and temperature, respectively, defines the Brownian orientational relaxation time $\tau=\left(2 D_{r}\right)^{-1}$. The vorticity $\boldsymbol{\Omega}$ and the symmetric velocity gradient $\boldsymbol{D}$ of the velocity field $\boldsymbol{v}(\boldsymbol{r})$ are defined as $\boldsymbol{\Omega}=\boldsymbol{\nabla} \times \boldsymbol{v} / 2$ and $\boldsymbol{D}=[\boldsymbol{\nabla} \boldsymbol{v}$ $\left.+(\boldsymbol{\nabla} \boldsymbol{v})^{\mathrm{T}}\right] / 2$. Finally, the so-called shape factor $B$ is defined 
as $B=\left(r^{2}-1\right) /\left(r^{2}+1\right)$. In the case of spherical particles, $r$ $=1, B=0$, Eq. (1) reduces to the kinetic equation proposed in Ref. [4].

In the following, we consider the case of a local magnetic field $\boldsymbol{H}$ where the local potential $V$ is given by

$$
V(\boldsymbol{u})=-\boldsymbol{\mu} \cdot \boldsymbol{H}=-k_{B} T \boldsymbol{u} \cdot \boldsymbol{h} .
$$

In Eq. (2), we have defined the dimensionless magnetic field $\boldsymbol{h}=\mu \boldsymbol{H} / k_{B} T$. For later use, we define the amplitude $h$ of $\boldsymbol{h}$ (Langevin parameter) and the unit vector in the direction of the magnetic field $\hat{\boldsymbol{h}}, \boldsymbol{h}=h \hat{\boldsymbol{h}}$. The stationary solution to Eq. (1) in the absence of flow gradients is given by the Boltzmann distribution

$$
f_{0}(\boldsymbol{u})=\frac{h}{4 \pi \sinh (h)} \exp (\boldsymbol{h} \cdot \boldsymbol{u})
$$

The macroscopic magnetization is given by $\boldsymbol{M}=M_{\mathrm{sat}}\langle\boldsymbol{u}\rangle$, where we have defined the saturation magnetization $M_{\text {sat }}$ $=n \mu$ and $\langle\bullet\rangle=\int d^{2} u \bullet f(\boldsymbol{u} ; t)$ denotes averages with respect to the distribution function $f$. In the stationary state (3), the magnetization is therefore $\boldsymbol{M}_{0}=M_{\text {sat }} L_{1}(h) \hat{\boldsymbol{h}}$, where $L_{1}(x)$ $=\operatorname{coth}(x)-x^{-1}$ is the Langevin function.

From the kinetic equation the following moment equations are derived by multiplying Eq. (1) with $\boldsymbol{u}$ and $\boldsymbol{u} \boldsymbol{u}$, respectively, and subsequent integration over $\boldsymbol{u}$. The result reads $[6,11]$

$$
\begin{aligned}
\tau \partial_{t}\langle\boldsymbol{u}\rangle+\langle\boldsymbol{u}\rangle= & \tau \boldsymbol{\Omega} \times\langle\boldsymbol{u}\rangle+B \tau(\boldsymbol{D} \cdot\langle\boldsymbol{u}\rangle-\langle\boldsymbol{u} \boldsymbol{u} \boldsymbol{u}\rangle: \boldsymbol{D}) \\
& +\frac{1}{2}(\boldsymbol{h}-\langle\boldsymbol{u} \boldsymbol{u}\rangle \cdot \boldsymbol{h})
\end{aligned}
$$

and

$$
\begin{aligned}
\tau \partial_{t}\langle\boldsymbol{u} \boldsymbol{u}\rangle+3\left\langle\boldsymbol{u} \boldsymbol{u}-\frac{1}{3} \mathbf{1}\right\rangle= & \tau \boldsymbol{W} \cdot\langle\boldsymbol{u} \boldsymbol{u}\rangle-\langle\boldsymbol{u} \boldsymbol{u}\rangle \cdot \tau \boldsymbol{W}+B \tau(\boldsymbol{D} \cdot\langle\boldsymbol{u} \boldsymbol{u}\rangle \\
& +\langle\boldsymbol{u} \boldsymbol{u}\rangle \cdot \boldsymbol{D}-2\langle\boldsymbol{u} \boldsymbol{u} \boldsymbol{u} \boldsymbol{u}\rangle: \boldsymbol{D})+\frac{1}{2}(\boldsymbol{h}\langle\boldsymbol{u}\rangle \\
& +\langle\boldsymbol{u}\rangle \boldsymbol{h}-2\langle\boldsymbol{u} \boldsymbol{u} \boldsymbol{u}\rangle \cdot \boldsymbol{h}) .
\end{aligned}
$$

The hydrodynamic stress tensor $\boldsymbol{T}$ for an incompressible dilute suspension of rigid ferromagnetic ellipsoids can be decomposed into its antisymmetric part

$$
\boldsymbol{T}^{a}=\frac{n k_{B} T}{2}(\boldsymbol{h}\langle\boldsymbol{u}\rangle-\langle\boldsymbol{u}\rangle \boldsymbol{h}),
$$

and its symmetric part $\boldsymbol{T}^{s}$ (see, e.g., Refs. [2,6,11]). The latter can be expressed with the help of Eq. (5) as

$$
\begin{aligned}
\boldsymbol{T}^{s}= & 2 \eta_{\mathrm{s}}\left(1+5 \phi Q_{1}\right) \boldsymbol{D}+5 \eta_{\mathrm{s}} \phi\left\{2 Q_{3}(\boldsymbol{D} \cdot\langle\boldsymbol{u} \boldsymbol{u}\rangle+\langle\boldsymbol{u} \boldsymbol{u}\rangle \cdot \boldsymbol{D})\right. \\
& \left.-Q_{23}\langle\boldsymbol{u} \boldsymbol{u} \boldsymbol{u} \boldsymbol{u}\rangle: \boldsymbol{D}+Q_{0}\left(\boldsymbol{W} \cdot\langle\boldsymbol{u} \boldsymbol{u}\rangle-\langle\boldsymbol{u} \boldsymbol{u}\rangle \cdot \boldsymbol{W}-\partial_{t}\langle\boldsymbol{u} \boldsymbol{u}\rangle\right)\right\} .
\end{aligned}
$$

The geometric coefficients $Q_{i}$ are defined in the Appendix A.

\section{APPROXIMATE SOLUTIONS OF THE KINETIC MODEL FOR WEAK FLOWS}

In the case of weak velocity gradients, the symmetric part of the stress tensor $\boldsymbol{T}^{s}$ is well approximated by

$$
\begin{aligned}
\boldsymbol{T}^{s}= & 2 \eta_{s}\left(1+5 \phi Q_{1}\right) \boldsymbol{D}+5 \eta_{s} \phi\left\{2 Q_{3}\left(\boldsymbol{D} \cdot\langle\boldsymbol{u} \boldsymbol{u}\rangle_{0}+\langle\boldsymbol{u} \boldsymbol{u}\rangle_{0} \cdot \boldsymbol{D}\right)\right. \\
& -Q_{23}\langle\boldsymbol{u} \boldsymbol{u} \boldsymbol{u} \boldsymbol{u}\rangle_{0}: \boldsymbol{D}+Q_{0}\left(\boldsymbol{W} \cdot\langle\boldsymbol{u} \boldsymbol{u}\rangle_{0}\right. \\
& \left.\left.-\langle\boldsymbol{u} \boldsymbol{u}\rangle_{0} \cdot \boldsymbol{W}-\partial_{t}\langle\boldsymbol{u} \boldsymbol{u}\rangle\right)\right\}
\end{aligned}
$$

where $\langle\bullet\rangle_{0}$ denotes averages with the equilibrium distribution function $f_{0}$. Thus, in order to evaluate the full stress tensor, knowledge of the moments $\langle\boldsymbol{u}\rangle$ and $\partial_{t}\langle\boldsymbol{u} \boldsymbol{u}\rangle$ is neccessary. From Eqs. (4) and (5), we observe that the moment equations form a hierarchy and that it is impossible to arrive rigorously at a closed set of equations for low order moments. This is true, in particular, for the magnetization and the hydrodynamic stress tensor. This so-called closure problem occurs in many branches of statistical physics and an enormous amount of closure approximations have been proposed in the literature [2]. In this contribution, we discuss two particular closure approximations that have been proposed in Refs. [6] and [4], respectively.

\section{A. Effective field approximation}

The effective field approximation (EFA) was introduced in Ref. [4] to solve the closure problem for the kinetic equation (1) in the case of spherical particles $(B=0)$. The EFA, which can be interpreted as the quasiequilibrium approximation for the lowest order moment of $f$ (see Ref. [11]), can also be applied to the case of rigid ellipsoidal particles with $B$ $\neq 0$. Within the EFA, the nonequilibrium distribution $f$ is assumed to be of the equilibrium form (3) but with the magnetic field $\boldsymbol{h}$ replaced by an effective field $\boldsymbol{\xi}_{e}$. Splitting the effective field $\boldsymbol{\xi}_{e}=\xi_{e} \hat{\boldsymbol{\xi}}_{e}$ into its norm $\xi_{e}$ and unit vector $\hat{\boldsymbol{\xi}}_{e}$, Eq. (4) becomes within the EFA

$$
\begin{aligned}
\tau \frac{d \xi_{e}}{d t}= & \left(\frac{d}{d \xi_{e}} \ln L_{1}\left(\xi_{e}\right)\right)^{-1}\left(3 B \frac{L_{2}\left(\xi_{e}\right)}{\xi_{e} L_{1}\left(\xi_{e}\right)} \tau \boldsymbol{D}: \hat{\boldsymbol{\xi}}_{e} \hat{\boldsymbol{\xi}}_{e}\right. \\
& \left.+\frac{h}{\xi_{e}} \hat{\boldsymbol{h}} \cdot \hat{\boldsymbol{\xi}}_{e}-1\right)
\end{aligned}
$$

and

$$
\begin{aligned}
\tau \frac{d \hat{\boldsymbol{\xi}}_{e}}{d t}= & \tau \boldsymbol{\Omega} \times \hat{\boldsymbol{\xi}}_{e}+B \alpha\left(\xi_{e}\right)\left(\boldsymbol{D} \cdot \hat{\boldsymbol{\xi}}_{e}-\hat{\boldsymbol{\xi}}_{e} \hat{\boldsymbol{\xi}}_{e} \hat{\boldsymbol{\xi}}_{e}: \boldsymbol{D}\right) \\
& +\frac{1}{2}\left(\frac{1}{L_{1}\left(\xi_{e}\right)}-\frac{1}{\xi_{e}}\right)\left(\boldsymbol{h}-\hat{\boldsymbol{\xi}}_{e} \hat{\boldsymbol{\xi}}_{e} \cdot \boldsymbol{h}\right),
\end{aligned}
$$

where $\alpha(x)=1-2 L_{2}(x) /\left[x L_{1}(x)\right]$. Functions $L_{j}(x)$ are defined recursively by $L_{j+1}(x)=L_{j-1}(x)-(2 j+1) L_{j}(x) / x$ with $L_{0}(x) \equiv 1$ and $L_{1}$ is the Langevin function [2]. For small amplitude oscillatory flow with frequency $\omega$ and constant magnetic field $\boldsymbol{h}$, explicit expressions for the resulting effective field may be obtained. Consider small deviations of the effective field from the equilibrium value, $\Delta \boldsymbol{\xi}_{e}=\boldsymbol{\xi}_{e}-\boldsymbol{h}$, 
$\Delta \xi_{e}=\xi_{e}-h$ with $\left|\Delta \xi_{e}\right| \ll h$ and let $\Delta \hat{\boldsymbol{\xi}}_{e}$ denote the direction of the off-equilibrium magnetization perpendicular to applied magnetic field, $\Delta \hat{\boldsymbol{\xi}}_{e} \cdot \hat{\boldsymbol{h}}=0$. Keeping only lowest order terms in the deviation from equilibrium, $\boldsymbol{\xi}_{e}=\boldsymbol{h}+\Delta \xi_{e} \hat{\boldsymbol{h}}+h \Delta \hat{\boldsymbol{\xi}}_{e}$, the nonequilibrium distribution function simplifies to

$$
f_{e}(\boldsymbol{u})=f_{0}(\boldsymbol{u})\left[1+\Delta \boldsymbol{\xi}_{e} \cdot \boldsymbol{u}-\Delta \xi_{e} L_{1}(h)\right] .
$$

It is readily verified that $f_{e}$, Eq. (11), is normalized, $\int d^{2} u f_{e}(\boldsymbol{u})=1$. Equations (9) and (10) become, with the help of Eq. (11) and linearization in the deviation from equilibrium,

$$
\begin{gathered}
\Delta \xi_{e}(t)=\left(1+i \tau \omega \frac{h L_{1}^{\prime}(h)}{L_{1}(h)}\right)^{-1} 3 B \frac{L_{2}(h)}{L_{1}(h)} \tau \boldsymbol{D}(t): \hat{\boldsymbol{h}} \hat{\boldsymbol{h}}, \\
\Delta \hat{\boldsymbol{\xi}}_{e}(t)=[1+i \tau \omega \beta(h)]^{-1} \beta(h) \boldsymbol{e}(t),
\end{gathered}
$$

where

$$
\boldsymbol{e}(t)=\tau \boldsymbol{\Omega}(t) \times \hat{\boldsymbol{h}}+B \tau \alpha(h)[\boldsymbol{D}(t) \cdot \hat{\boldsymbol{h}}-\hat{\boldsymbol{h}} \hat{\boldsymbol{h}} \hat{\boldsymbol{h}}: \boldsymbol{D}(t)],
$$

and $\beta(x)=2 L_{1}(x) /\left[x-L_{1}(x)\right]$. In Eqs. (12) and (13), we have assumed that, after the oscillatory flow has been applied for a sufficiently long time, the effective field oscillates with the same frequency $\omega$ as the applied flow. The predictions of the EFA in case of weak stationary flow are obtained from Eqs. (12)-(14) for $\omega=0$.

\section{B. Linearized moment expansion}

In order to solve the closure problem, the authors of Ref. [6] expand the distribution function $f$ around the equilibrium distribution

$$
f(\boldsymbol{u})=f_{0}(\boldsymbol{u})\left[1+\boldsymbol{a} \cdot\left(\boldsymbol{u}-\langle\boldsymbol{u}\rangle_{0}\right)+\boldsymbol{b}:\left(\boldsymbol{u} \boldsymbol{u}-\langle\boldsymbol{u} \boldsymbol{u}\rangle_{0}\right)\right],
$$

with unknown coefficients $\boldsymbol{a}$ and $\boldsymbol{b}$. The normalization of $f$ is ensured since $\langle\bullet\rangle_{0}$ denotes averages with the equilibrium distribution function (3). Ansatz (15) is valid for small Péclet number flows, where $\boldsymbol{a}$ and $\boldsymbol{b}$ are first order in the velocity gradient. Note that the linearized EFA, Eq. (11), is obtained as a special case of Eq. (15) for $\boldsymbol{b}=\mathbf{0}$, since within the EFA the corresponding term is second order in the deviation from equilibrium. The linearized moment expansion (LME) assumes that the coefficients $\boldsymbol{a}$ and $\boldsymbol{b}$ are independent of the orientation $\boldsymbol{u}$. Inserting the ansatz (15) into the moment equations (4) and (5) and linearization in the velocity gradients leads to the following system of linear equations for the unknown coefficients $\boldsymbol{a}$ and $\boldsymbol{b}$ :

$$
\begin{aligned}
& \boldsymbol{A}^{(1,1)} \cdot \boldsymbol{a}+\boldsymbol{A}^{(1,2)}: \boldsymbol{b}=\boldsymbol{E}^{(1),}, \\
& \boldsymbol{A}^{(2,1)} \cdot \boldsymbol{a}+\boldsymbol{A}^{(2,2)}: \boldsymbol{b}=\boldsymbol{E}^{(2),}
\end{aligned}
$$

where $\boldsymbol{E}^{(1)}$ and $\boldsymbol{E}^{(2)}$ denote the right-hand side of Eqs. (4) and (5) for $h=0$, respectively, if all averages are performed with the equilibrium distribution function $f_{0}$. In Eq. (16), we have defined the quantities

$$
\begin{gathered}
\boldsymbol{A}^{(1, j)}=\boldsymbol{C}^{(1, j)}\left(1+\frac{d}{d t}\right)+\frac{1}{2} \boldsymbol{h} \cdot \boldsymbol{C}^{(2, j)}, \\
\boldsymbol{A}^{(2, j)}=\boldsymbol{C}^{(2, j)}\left(3+\frac{d}{d t}\right)-\left(\boldsymbol{h} \boldsymbol{C}^{(1, j)}\right)_{\mathrm{sym}}+\boldsymbol{h} \cdot \boldsymbol{C}^{(3, j)},
\end{gathered}
$$

for $j=1,2$, and the correlation functions of the order of $j$ $+k$ given by

$$
\boldsymbol{C}^{(j, k)} \equiv\langle\underbrace{\boldsymbol{u} \ldots \boldsymbol{u} \boldsymbol{u} \ldots \boldsymbol{u}}_{j}\rangle_{0}-\langle\underbrace{\boldsymbol{u} \ldots \boldsymbol{u}}_{j}\rangle_{0}\langle\underbrace{\boldsymbol{u} \ldots \boldsymbol{u}}_{k}\rangle_{0} .
$$

The notation $(\bullet)_{\text {sym }}$ implies symmetrization after multiplication with $\boldsymbol{a}$ and $\boldsymbol{b}$, respectively. We assume that the moments $\langle\boldsymbol{u}\rangle$ and $\langle\boldsymbol{u} \boldsymbol{u}\rangle$ oscillate with the same frequency $\boldsymbol{\omega}$ as the applied flow. Then, the differential operators $\boldsymbol{A}^{(i, j)}$ become ordinary, complex matrices and the resulting algebraic system of linear equations (16) can be solved for the coefficients $\boldsymbol{a}$ and $\boldsymbol{b}$ by matrix inversion.

\section{COMPARISON TO BROWNIAN DYNAMICS SIMULATION}

In the sequel, we consider exclusively the case of plane Couette flow, $\boldsymbol{v}(\boldsymbol{r} ; t)=(\dot{\gamma}(t) y, 0,0)$, where $\dot{\gamma}(t)$ denotes the shear rate. The case of stationary $\dot{\gamma}(t)=\dot{\gamma}_{0}$ and oscillatory $\dot{\gamma}(t)=\dot{\gamma}_{0} e^{i \omega t}$ flow is considered separately. The Péclet number is defined as $\mathrm{Pe}=\tau \dot{\gamma}_{0}$. Since the approximations introduced in Sec. III apply for weak flows, we choose $\mathrm{Pe}=0.1$ in the sequel. We here consider magnetic fields that are oriented either in flow or in gradient direction. For magnetic fields that are oriented in the vorticity direction of the flow see Ref. [11]. Two representative values for the axis ratio $r$ have been chosen, $r=2$ and $r=5$. While $r=1$ corresponds to spherical particles that have been studied previously in Refs. [12,13], values of $r \geqslant 5$ give very similar results since in this case the shape factor $B$ is close to one.

In order to discuss the quality and range of validity of the approximations presented in Sec. III, we compare those predictions to the numerical solution of the kinetic equation (1). To this end, we perform Brownian dynamics (BD) simulations of the corresponding Itô stochastic differential equation of the stochastic process $\boldsymbol{U}_{t}$ [11],

$$
d \boldsymbol{U}_{t}=\boldsymbol{P}_{t} \cdot\left[\left(\boldsymbol{\Omega} \times \boldsymbol{U}_{t}+B \boldsymbol{D} \cdot \boldsymbol{U}_{t}+D_{r} \boldsymbol{h}\right) d t+d \boldsymbol{W}_{t}\right]-D_{r} \boldsymbol{U}_{t} d t,
$$

where $\boldsymbol{P}_{t}=\mathbf{1}-\boldsymbol{U}_{t} \boldsymbol{U}_{t}$ and $\boldsymbol{W}_{t}$ is a three-dimensional Wiener process. By construction $d\left(\boldsymbol{U}_{t}^{2}\right)=0$ due to Itô's formula. Ensemble averages of arbitrary functions $A\left(\boldsymbol{U}_{t}\right)$, where $\boldsymbol{U}_{t}$ are solutions to Eq. (20), converge with increasing ensemble to averages $\langle A(\boldsymbol{u})\rangle$, with the correct distribution function. In order to achieve accurate averages, an ensemble of $N=10^{5}$ unit vectors $\boldsymbol{U}_{t}$ is evolved. As done in Ref. [11], we use a weak first-order scheme to integrate Eq. (20) numerically, keeping the normalization fixed, $\boldsymbol{U}_{t}^{2}=1$. 

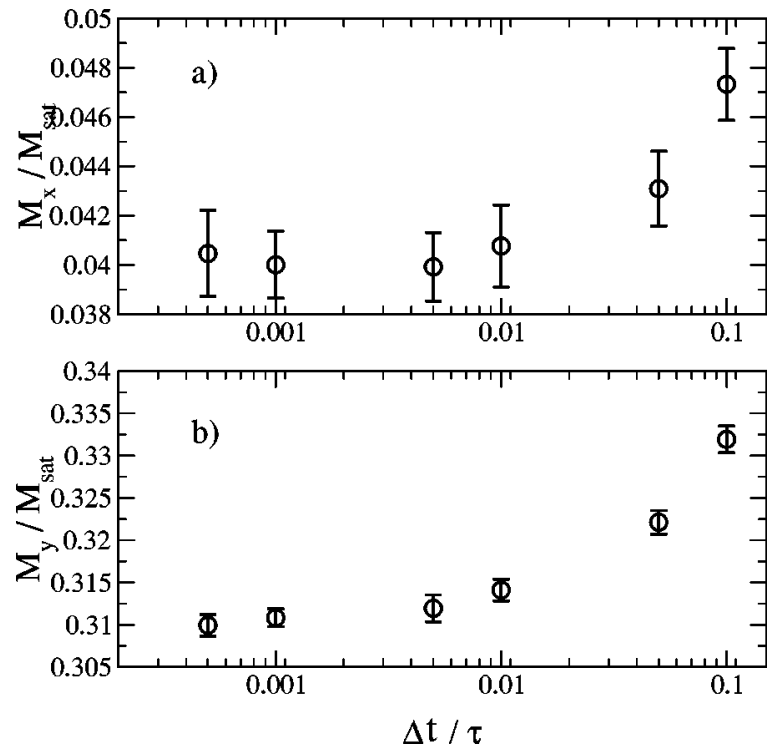

FIG. 1. The dependence of the stationary magnetization (a) $M_{x} / M_{\text {sat }}$, (b) $M_{y} / M_{\text {sat }}$, on the time step $\Delta t$ of the numerical integration for the dimensionless magnetic field $\boldsymbol{h}=(0,1,0)$. A plane Couette flow with constant Péclet number $\mathrm{Pe}=0.1$ was considered with $x$ the flow direction and $y$ the gradient direction, respectively. The value of the axis ratio of the ellipsoid was chosen as $r=5$. Symbols represent time averages of the result of the BD simulations, the errorbars show the corresponding standard deviations.

\section{A. Stationary flow}

First, consider the case of stationary plane Couette flow, $\boldsymbol{v}(\boldsymbol{r})=\left(\dot{\gamma}_{0} y, 0,0\right)$, with constant shear rate $\dot{\gamma}_{0}$. The BD simulations, Eq. (20), were started either from the equilibrium, Eq. (3), or from a perfect oriented distribution and integrated for a fixed time step $\Delta t$. The same stationary state was attained after $\approx 5 \tau$, independent of the initial configuration. Results for the stationary values were extracted as time averages for $10 \tau \leqslant t \leqslant 20 \tau$ at times $0.1 \tau$. Error bars for the simulation results are determined by the standard deviation of the mean.

Figure 1 shows the influence of the time step $\Delta t$ on the simulation results for the stationary magnetization for $\mathrm{Pe}$ $=0.1$ and magnetic field oriented in gradient direction with $h=1$. The axis ratio of the ellipsoid was chosen as $r=5$. From Fig. 1, we notice that the results are more or less independent of the time step for $\Delta t / \tau \lesssim 10^{-2}$. Thus, we choose a time step $\Delta t=10^{-3} \tau$ in the sequel in order to obtain accurate stationary results.

The shear viscosity $\eta_{y x}$ is defined as $\eta_{y x}=T_{y x} / \dot{\gamma}_{0}$, where the hydrodynamic stress was evaluated from Eqs. (6) and (7). The symmetric and antisymmetric part of the stress tensor, Eqs. (6) and (7), define symmetric and antisymmetric contributions to the shear viscosity $\eta_{y x}$,

$$
\eta_{y x}=\eta_{y x}^{s}+\eta_{y x}^{a}
$$

For weak flow, $\tau \dot{\gamma}_{0} \ll 1$, the symmetric contribution to the shear viscosity $\eta_{y x}^{s}$ can be obtained from Eq. (8) in the stationary state,

$$
\begin{aligned}
\eta_{y x}^{s}= & \eta_{s}+\frac{5}{2} \eta_{s} \phi\left\{2 Q_{1}+4 Q_{3} \frac{L_{1}(h)}{h}-2 Q_{23} \frac{L_{2}(h)}{h^{2}}\right. \\
& +2\left(Q_{3} L_{2}(h)-Q_{23} \frac{L_{3}(h)}{h}\right)\left(\hat{h}_{x}^{2}+\hat{h}_{y}^{2}\right) \\
& \left.+Q_{0} L_{2}(h)\left(\hat{h}_{y}^{2}-\hat{h}_{x}^{2}\right)-2 Q_{23} L_{4}(h) \hat{h}_{x}^{2} \hat{h}_{y}^{2}\right\} .
\end{aligned}
$$

In order to obtain the resulting shear viscosity (21), expressions for the off-equilibrium magnetization have to be found.

The predictions of the EFA for stationary Couette flow are obtained from Eqs. (12) $-(14)$ by setting $\omega=0$. One obtains for the stationary off-equilibrium magnetization

$$
\frac{\boldsymbol{M}^{\mathrm{EFA}}-\boldsymbol{M}_{0}}{M_{\mathrm{sat}}}=3 B \mathrm{Pe} \frac{L_{1}^{\prime}(h) L_{2}(h)}{L_{1}(h)} \hat{h}_{x} \hat{h}_{y} \hat{\boldsymbol{h}}+\frac{2 L_{1}^{2}(h)}{h-L_{1}(h)} \boldsymbol{e},
$$

where the vector $\boldsymbol{e}$, Eq. (14), takes the form

$$
\boldsymbol{e}=\frac{1}{2} \operatorname{Pe}\left(\begin{array}{c}
\hat{h}_{y}\left\{1+B \alpha(h)\left[1-2 \hat{h}_{x}^{2}\right]\right\} \\
\hat{h}_{x}\left\{-1+B \alpha(h)\left[1-2 \hat{h}_{y}^{2}\right]\right\} \\
-2 B \alpha(h) \hat{h}_{x} \hat{h}_{y} \hat{h}_{z}
\end{array}\right) .
$$

From Eqs. (23) and (24), the asymptotic behavior of the off-equilibrium magnetization, if the magnetic field is oriented in flow direction $(p=1)$ or in gradient direction $(p$ $=2$ ), is given by

$$
M_{\nu}^{\mathrm{EFA}} / M_{\mathrm{sat}}=\operatorname{Pe}\left\{\begin{array}{cc}
\frac{h}{3}\left[(-1)^{p}+\frac{3}{5} B\right] & \text { for } h \rightarrow 0 \\
\frac{2}{h}\left[(-1)^{p}+B\right] & \text { for } h \rightarrow \infty,
\end{array}\right.
$$

where $\nu=x$ for $p=2$ and $\nu=y$ for $p=1$. Thus, within the EFA, the off-equilibrium magnetization increases linearly with $h$ for $h \ll 1$, the slope being related to the shape factor $B$ of the colloidal particles. For $h \gg 1$, the off-equilibrium magnetization decays as $h^{-1}$ where the prefactor depends on $B$. From magnetization (23), the antisymmetric contribution to the shear viscosity within the EFA is easily obtained,

$$
\eta_{y x}^{a, \text { EFA }}=\frac{\tau n k_{B} T}{2} \frac{h L_{1}^{2}(h)}{h-L_{1}(h)} \hat{g}_{y x}^{(2)}(h),
$$

where

$$
\hat{g}_{y x}^{(2)}(h)=\hat{h}_{x}^{2}+\hat{h}_{y}^{2}+B \alpha(h)\left(\hat{h}_{y}^{2}-\hat{h}_{x}^{2}\right)
$$

The predictions of the LME are obtained by solving the system of Eqs. (16)-(18) for the coefficients $\boldsymbol{a}$ and $\boldsymbol{b}$. If the magnetic field is oriented in flow (gradient) direction, only the components $a_{y}\left(a_{x}\right)$ and $b_{x y}$ are nonzero. In the stationary state, the differential operators $\boldsymbol{A}_{i j}$, Eqs. (17) and (18), become ordinary matrices and the coefficients $\boldsymbol{a}$ and $\boldsymbol{b}$ are obtained by inversion of a $2 \times 2$ matrix [6]. Having obtained 


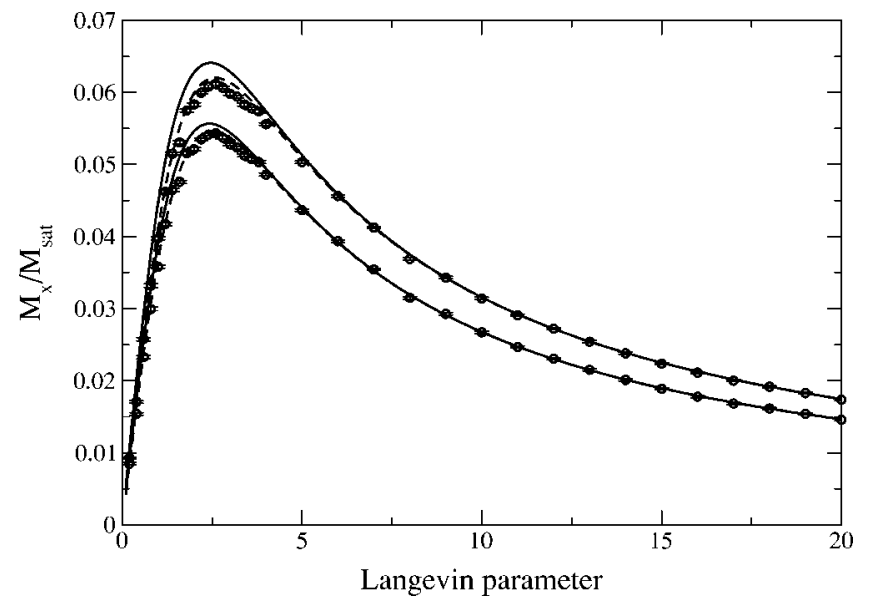

FIG. 2. Stationary off-equilibrium magnetization $M_{x} / M_{\text {sat }}$ in plane Couette flow as a function of the Langevin parameter $h$. The magnetic field was oriented in gradient direction. The Péclet number was chosen as $\mathrm{Pe}=0.1$. Symbols represent the result of the BD simulations. The EFA (full line) and LME (dashed line) are indistinguishable within the resolution of the figure. The value of the axis ratio of the ellipsoid was chosen as $r=2$ for the lower and $r$ $=5$ for the upper curves.

the coefficients $\boldsymbol{a}$ and $\boldsymbol{b}$, the antisymmetric contribution to the shear viscosity is found by

$$
\begin{aligned}
\eta_{y x}^{a, \mathrm{LME}}= & \frac{n k_{B} T h}{2 \dot{\gamma}_{0}}\left\{\frac{L_{1}(h)}{h}\left(a_{x} \hat{h}_{y}-a_{y} \hat{h}_{x}\right)\right. \\
& \left.+2 \frac{L_{2}(h)}{h}\left(b_{x \nu} \hat{h}_{\nu} \hat{h}_{y}-b_{y \nu} \hat{h}_{\nu} \hat{h}_{x}\right)\right\} .
\end{aligned}
$$

Figure 2 shows the stationary off-equilibrium magnetization $M_{x}=M_{\text {sat }}\left\langle u_{x}\right\rangle$ for magnetic fields oriented in gradient direction, $\boldsymbol{h}=(0, h, 0)$. The Péclet number was chosen as $\mathrm{Pe}$ $=0.1$. From Fig. 2, we observe that both, the EFA and LME predict the increase of $M_{x}$ with increasing $h$ for $h<h_{c}$ and the slow decay for $h>h_{c}$, where the value of the critical dimensionless magnetic field is $h_{c} \approx 2.5$ and 2.6 for axis ratios $r=2$ and $r=5$, respectively. While the EFA slightly overpredict the values of $M_{x}$ near $h_{c}$, the LME is almost indistinguishable from the results of the BD simulation for all values of $h$.

Figure 3 shows the relative change of the shear viscosity $\Delta_{y x}=\left[\eta_{y x}-\eta_{y x}^{s}(0)\right] / \tau n k_{B} T$ with and without magnetic field as a function of $h$. Here, $\eta_{y x}^{s}(0)$ denotes the symmetric shear viscosity (22) evaluated at $h=0$. Same as in Fig. 2, the magnetic field is oriented in gradient direction and the Péclet number was chosen as $P e=0.1$. From Fig. 3, we observe that the agreement of both approximations with the result of the BD simulations is excellent for axis ratios $r=2$ and $r=5$ and all values of $h$ considered.

Now we consider the case where the magnetic field is oriented in flow direction. The EFA predicts that in this case the off-equilibrium magnetization $M_{y}$ points in the direction of decreasing velocity, see Eq. (25). In Fig. 4, we show the reduced off-equilibrium magnetization $M_{y} / M_{\text {sat }}$ as a function of $h$, where the magnetic field is oriented in flow direc-

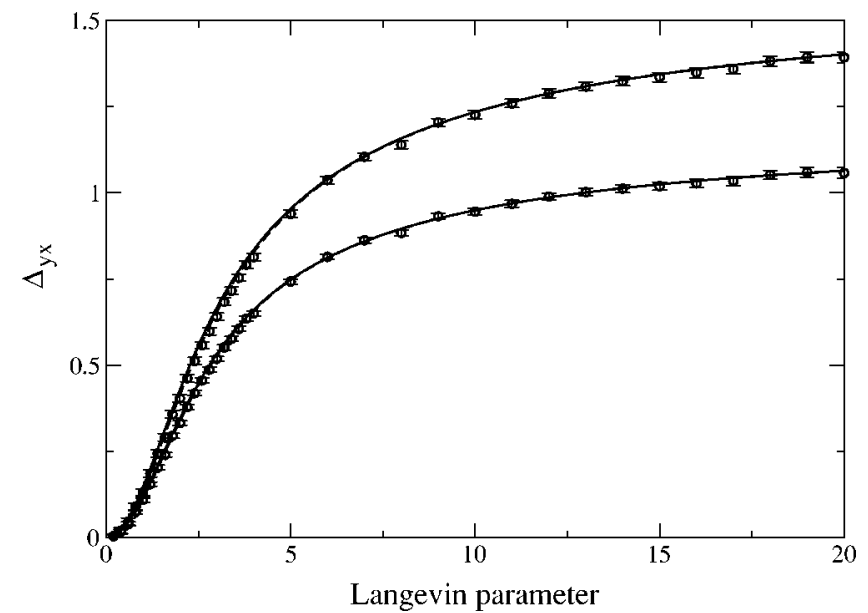

FIG. 3. Stationary relative change of shear viscosity $\Delta_{y x}$ in plane Couette flow as a function of the Langevin parameter $h$. The magnetic field was oriented in gradient direction. The Péclet number was chosen as $\mathrm{Pe}=0.1$. Symbols represent the result of the BD simulations, full line corresponds to the EFA, dashed line to the LME. The value of the axis ratio of the ellipsoid was chosen as $r$ $=2$ for the lower and $r=5$ for the upper curves.

tion. Indeed, the results of the BD simulations agree with the predictions of the EFA quantitatively for weak and strong fields. However, the values of $M_{y}$ for intermediate values of $h$ are not accurately described by the EFA. The LME on the contrary provides a much better description of the BD results for all values of $h$.

Figure 5 shows the relative change of the shear viscosity $\Delta_{y x}(h)$ for the same conditions as in Fig. 4. We observe an increase of the effective viscosity for all values of $h$ for axis ratio $r=2$, while in case $r=5$ the effective viscosity is decreased for sufficiently strong magnetic fields. From Fig. 5, we observe that the EFA is in qualitative agreement with the results of the BD simulation and provides quantitative accu-

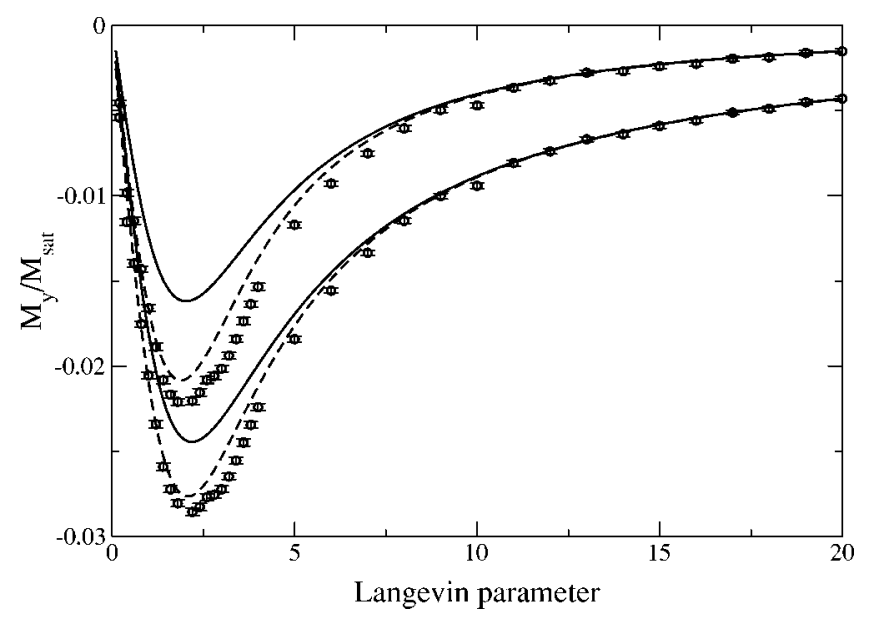

FIG. 4. Stationary off-equilibrium magnetization $M_{y} / M_{\text {sat }}$ in plane Couette flow as a function of the Langevin parameter $h$. The magnetic field was oriented in flow direction. The Péclet number was chosen as $\mathrm{Pe}=0.1$. Symbols represent the result of the $\mathrm{BD}$ simulations, full line corresponds to the EFA, dashed line to the LME. The value of the axis ratio of the ellipsoid was chosen as $r$ $=2$ for the lower and $r=5$ for the upper curves. 


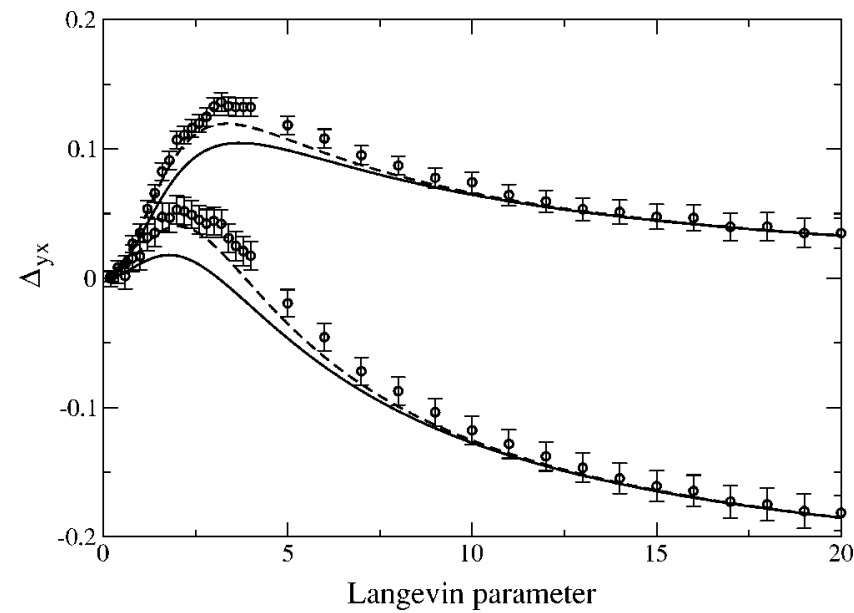

FIG. 5. Stationary relative change of shear viscosity $\Delta_{y x}$ in plane Couette flow as a function of the Langevin parameter $h$. The magnetic field was oriented in flow direction. The Péclet number was chosen as $\mathrm{Pe}=0.1$. Symbols represent the result of the BD simulations, full line corresponds to the EFA, dashed line to the LME. The value of the axis ratio of the ellipsoid was chosen as $r$ $=2$ for the upper and $r=5$ for the lower curves.

rate predictions in case of weak and strong magnetic fields. For intermediate values of $h$, however, the predictions of the EFA underestimate the results of the BD simulation. In this case again the LME provides an improvement compared to the EFA.

\section{B. Oscillatory flow}

Now, we consider the case of oscillatory Couette flow, $\boldsymbol{v}(\boldsymbol{r} ; t)=(\dot{\gamma}(t) y, 0,0)$, with oscillatory shear rate $\dot{\gamma}(t)$ $=\dot{\gamma}_{0} e^{i \omega t}$ and $\dot{\gamma}_{0}=\gamma_{0} \omega$. The BD simulations were performed for constant amplitude $\gamma_{0}$ for different frequencies, where the time step of integration was reduced for high oscillation frequencies $\omega$. After initial transient dynamics, an oscillatory stress response with frequency $\omega$ is observed. The effective complex shear viscosity $\eta_{y x}(\omega)$ is defined by $T_{y x}$ $=\eta_{y x}(\omega) \dot{\gamma}(t)$. Decomposing $\eta_{y x}$ into its real and imaginary part, $\eta_{y x}=\eta_{y x}^{\prime}-i \eta_{y x}^{\prime \prime}, \eta_{y x}^{\prime}$ and $\eta_{y x}^{\prime \prime}$ are determined from the in-phase and out-of-phase response of the stress. Errors in determining $\eta_{y x}^{\prime}$ and $\eta_{y x}^{\prime \prime}$ are estimated on the basis of the fit values for the amplitude and phase lag of the oscillatory stress response. Storage and loss moduli are related to the complex shear viscosity by $G^{\prime}(\omega)=\omega \eta_{y x}^{\prime \prime}(\omega)$ and $G^{\prime \prime}(\omega)$ $=\omega \eta_{y x}^{\prime}(\omega)$.

Decomposing the resulting time-dependent magnetization into $\boldsymbol{M}(t)=\boldsymbol{M}^{\prime}(t)-i \boldsymbol{M}^{\prime \prime}(t)$, the EFA predicts

$$
\begin{aligned}
& M_{\nu}^{\prime} / M_{\mathrm{sat}}=\frac{1}{2} \tau \dot{\gamma}(t)\left[(-1)^{p}+B \alpha(h)\right] \frac{\beta(h) L_{1}(h)}{1+(\tau \omega)^{2} \beta^{2}(h)}, \\
& M_{\nu}^{\prime \prime} / M_{\mathrm{sat}}=\frac{1}{2} \tau \dot{\gamma}(t)\left[(-1)^{p}+B \alpha(h)\right] \frac{\tau \omega \beta^{2}(h) L_{1}(h)}{1+(\tau \omega)^{2} \beta^{2}(h)} .
\end{aligned}
$$

As before, $\nu=x, p=2$ corresponds to the magnetic field that are oriented in gradient direction, while $\nu=y, p=1$ applies if the magnetic field is oriented in flow direction.

The result of the EFA for the real and imaginary part of the effective complex shear viscosity is

$$
\begin{aligned}
\eta_{y x}^{\prime}= & \eta_{y x}^{s}-\frac{5}{2} \eta_{s} \phi Q_{0} L_{2}(h) \frac{(\tau \omega)^{2} \beta^{2}(h)}{1+(\tau \omega)^{2} \beta^{2}(h)} \hat{g}_{y x}^{(1)}(h) \\
+ & \frac{\tau n k_{B} T}{4} \frac{h L_{1}(h) \beta(h)}{1+(\tau \omega)^{2} \beta^{2}(h)} \hat{g}_{y x}^{(2)}(h) \\
\eta_{y x}^{\prime \prime}= & \frac{5}{2} \eta_{s} \phi Q_{0} L_{2}(h) \frac{\tau \omega \beta(h)}{1+(\tau \omega)^{2} \beta^{2}(h)} \hat{g}_{y x}^{(1)}(h) \\
& +\frac{\tau n k_{B} T}{4} \frac{(\tau \omega) h L_{1}(h) \beta^{2}(h)}{1+(\tau \omega)^{2} \beta^{2}(h)} \hat{g}_{y x}^{(2)}(h)
\end{aligned}
$$

where

$$
\hat{g}_{y x}^{(1)}(h)=\hat{h}_{y}^{2}-\hat{h}_{x}^{2}+B \alpha(h)\left(\hat{h}_{x}^{2}-4 \hat{h}_{x}^{2} \hat{h}_{y}^{2}+\hat{h}_{y}^{2}\right) .
$$

For vanishing frequency, $\omega \rightarrow 0, \eta_{y x}^{\prime \prime} \rightarrow 0$ and $\eta_{y x}^{\prime}$ reduces to the stationary viscosity $\eta_{y x}$. The low and high frequency behavior is given by

$$
\begin{gathered}
\eta_{y x}^{\prime}(\omega)=\left\{\begin{array}{cc}
\eta_{y x}-a_{0}^{\prime}(\tau \omega)^{2} & \text { for } \tau \omega \ll 1 \\
a_{\infty}^{\prime} & \text { for } \tau \omega \gg 1,
\end{array}\right. \\
\eta_{y x}^{\prime \prime}(\omega)=\left\{\begin{array}{cc}
a_{0}^{\prime \prime} \tau \omega & \text { for } \tau \omega \ll 1 \\
a_{\infty}^{\prime \prime} /(\tau \omega) & \text { for } \tau \omega \gg 1,
\end{array}\right.
\end{gathered}
$$

where

$$
a_{0}^{\prime}=\frac{5}{2} \eta_{s} \phi Q_{0} L_{2} \beta^{2} \hat{g}_{y x}^{(1)}+\frac{\tau n k_{B} T}{4} h L_{1} \beta^{3} \hat{g}_{y x}^{(2)}
$$

$a_{0}^{\prime \prime}=a_{0}^{\prime} / \beta, a_{\infty}^{\prime \prime}=a_{0}^{\prime} / \beta^{3}$, and $a_{\infty}^{\prime}=\eta_{y x}^{\mathrm{s}}-(5 / 2) \eta_{\mathrm{s}} \phi Q_{0} L_{2} \hat{g}_{y x}^{(1)}$. In Figs. 6 and 7 , the dimensionless effective viscosities $\theta_{y x}^{\prime}$ $\equiv\left(\eta_{y x}^{\prime}-\eta_{s}\right) / \tau n k_{B} T$ and $\theta_{y x}^{\prime \prime} \equiv \eta_{y x}^{\prime \prime} / \tau n k_{B} T$ are shown as a function of the frequency $\omega$ of the applied shear flow. The magnetic field was oriented in gradient direction with the strength $h=1$. The axis ratios are $r=2$ and $r=5$, respectively. From Figs. 6 and 7, we observe that the EFA provides a good approximation for $\tau \omega<1$, while its accuracy for high frequencies is poor. The LME is found to be accurate for all values of $\tau \omega$ investigated here.

Figure 8 shows the dimensionless effective viscosities $\theta_{y x}^{\prime}$ and $\theta_{y x}^{\prime \prime}$ for the same conditions as in Fig. 7 but for a strong magnetic field, $h=10$. From Fig. 8, we deduce that in this case both approximations provide an accurate description of the $\mathrm{BD}$ results for all values of $\tau \omega$. In addition, the low frequency expansion, given by the first part of Eqs. (34) and (35), respectively, provide an acceptable description of the BD results for $\tau \omega \leqslant 2$. 


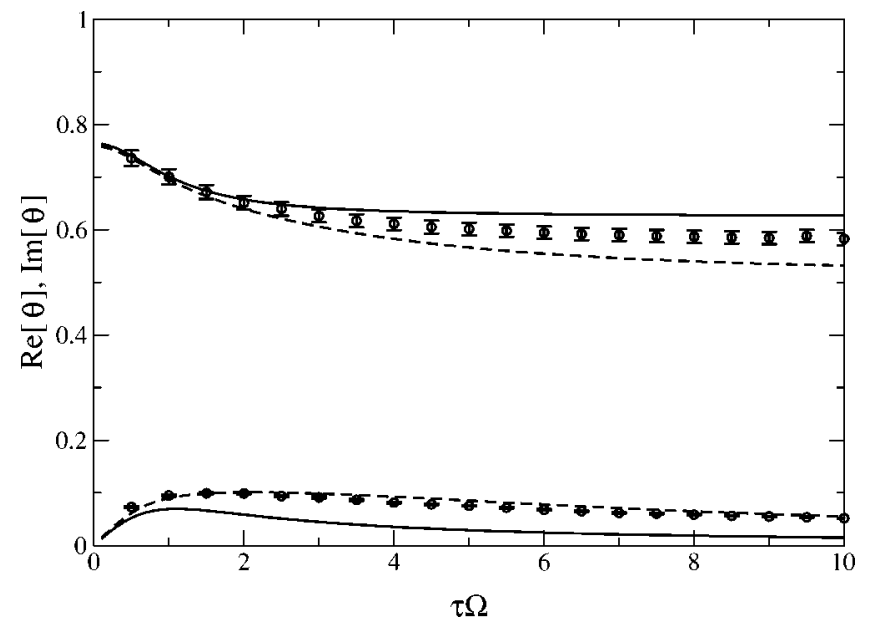

FIG. 6. Dimensionless complex effective viscosity in oscillatory shear flow as a function of the reduced frequency $\tau \omega$. The real part $\theta_{y x}^{\prime}$ corresponds to the upper and the imaginary part $\theta_{y x}^{\prime \prime}$ to the lower curves. The same conditions as in Fig. 2 were chosen with $r=2$ and $h=1$.

\section{CONCLUSIONS}

In the present contribution, we have compared the predictions of dynamic properties of different approximations to the kinetic model proposed in Ref. [6] to the result of Brownian dynamics simulation. We found that the effective field approximation and the linearized moment expansion provide very good approximations to the stationary offequilibrium magnetization as well as the stationary shear viscosity in case of weak Couette flow if the magnetic field is oriented in gradient direction. If the magnetic field is oriented in flow direction, the predictions of the EFA deviate strongly from the results of the BD simulation for intermediate values of the Langevin parameter $h$. The LME shows good agreement with the simulation data for the whole range of $h$. For small amplitude oscillatory Couette flow, we ob-

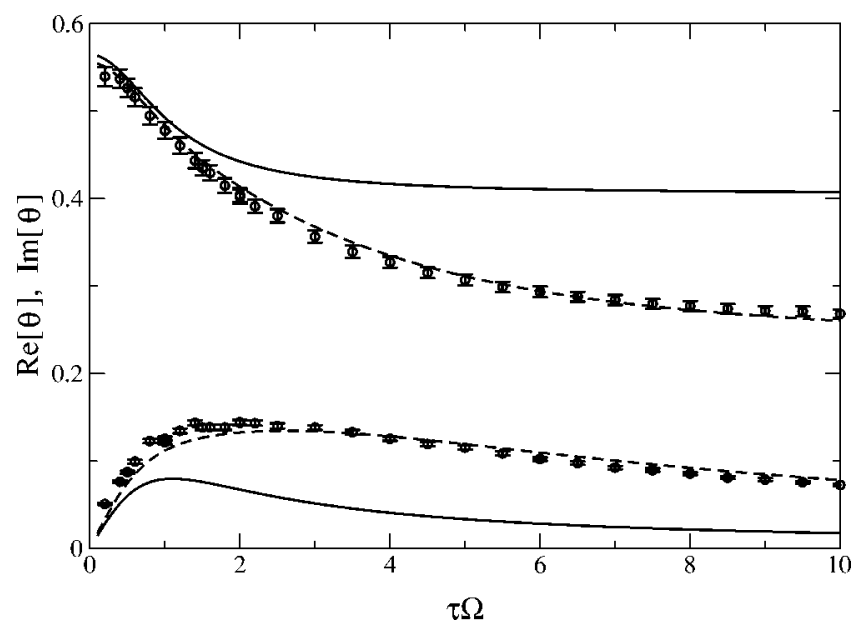

FIG. 7. Dimensionless complex effective viscosity in oscillatory shear flow as a function of the reduced frequency $\tau \omega$. The real part $\theta_{y x}^{\prime}$ corresponds to the upper and the imaginary part $\theta_{y x}^{\prime \prime}$ to the lower curves. The same conditions as in Fig. 2 were chosen with $r=5$ and $h=1$.

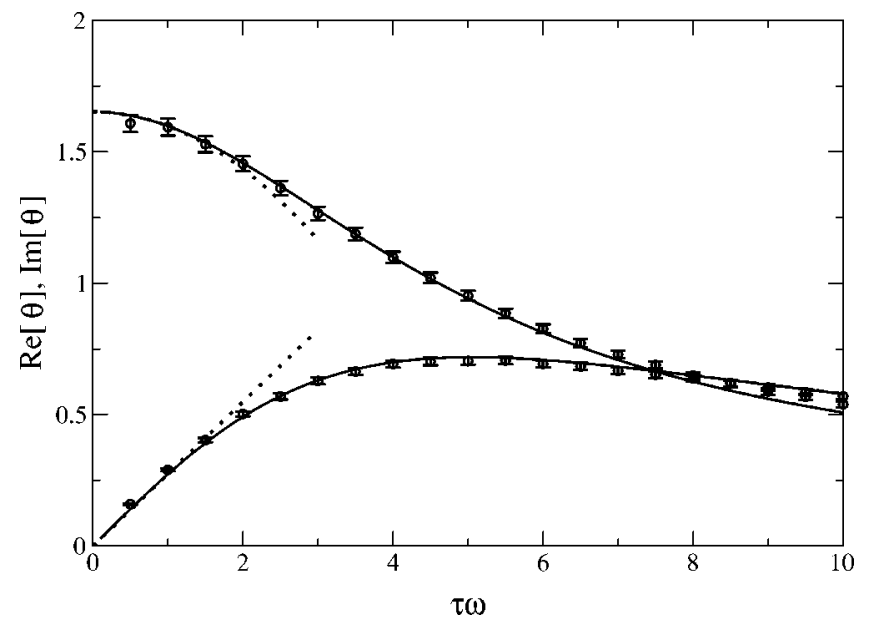

FIG. 8. Dimensionless complex effective viscosity in oscillatory shear flow as a function of the reduced frequency $\tau \omega$. The real part $\theta_{y x}^{\prime}$ corresponds to the upper and the imaginary part $\theta_{y x}^{\prime \prime}$ to the lower curves for small values of $\tau \omega$. The same conditions as in Fig. 2 were chosen with $r=5$ and $h=10$. The dotted lines correspond to the low frequency expansion, Eq. (34) and (35) for small $\tau \omega$.

serve that the predictions of the effective field approximation become less reliable with increasing frequency, while the predictions of the linearized moment expansion deviate only slightly over the whole frequency interval investigated here.

\section{ACKNOWLEDGMENTS}

Financial support provided by the Deutsche Forschungsgemeinschaft (DFG) under Grant No. HE 1100/6-3 is gratefully acknowledged. This work was partially supported also by grants of RFBR, Project Nos. 01-01-00058 and 03-0204001, Grant Nos. E02-3.2-164 and CRDR, REC-005.

\section{APPENDIX: GEOMETRIC COEFFICIENTS}

The geometric coefficients $Q_{i}$ depend only on the axis ratio $r$ of the ellipsoid and are given explicitly by [11]

$$
\begin{gathered}
Q_{0}=\frac{2\left(r^{2}-1\right)^{2}}{5 r^{2}\left(2 r^{2} \beta-\beta-1\right)}, \\
Q_{1}=\frac{4\left(r^{2}-1\right)^{2}}{5 r^{2}\left(3 \beta+2 r^{2}-5\right)}, \\
Q_{2}=\frac{2 Q_{1}}{3}\left[1-\frac{2 r^{2}+1-\left(4 r^{2}-1\right) \beta}{4\left(2 r^{2}+1\right) \beta-12}\right], \\
Q_{3}=Q_{1}\left[\frac{\left[r^{2}(\beta+1)-2\right]\left(3 \beta+2 r^{2}-5\right)}{4\left[\beta\left(2 r^{2}-1\right)-1\right]\left(r^{2}+2-3 r^{2} \beta\right)}-1\right],
\end{gathered}
$$

and

$$
Q_{23} \equiv 3 Q_{2}+4 Q_{3},
$$


for convenience, where

$$
\beta=\frac{1}{r \sqrt{\left|r^{2}-1\right|}} \times \begin{cases}\cosh ^{-1} r & \text { for } r>1 \\ \cos ^{-1} r & \text { for } r<1\end{cases}
$$

The rotational friction coefficient $\zeta_{r}$ is given by

$$
\zeta_{r}=10 \eta_{s} v \frac{Q_{0}}{B}
$$

where $v=\frac{4}{3} \pi a b^{2}$ is the volume of the ellipsoid. Equation (A4) corrects a misprint in Eqs. (B1) and (B3) of Ref. [11]. In Refs. [2,6], a different notation for the coefficients $Q_{i}$ is used, $\beta_{n}=5 \phi Q_{0}, \alpha_{n}=5 \phi Q_{1}, \zeta_{n}=5 \phi\left(2 Q_{3}-B Q_{0}\right), \chi_{n}=$ $-5 \phi\left(Q_{23}-2 B Q_{0}\right), \lambda_{n}=B$, and $n=r$.
[1] Ferrofluids. Magnetically Controllable Fluids and Their Applications, edited by S. Odenbach, Lecture Notes in Physics Vol. 594 (Springer, Berlin, 2002).

[2] E. Blums, A. Cebers, and M. M. Maiorov, Magnetic Fluids (Gruyter, Berlin, 1997).

[3] M. Kröger, P. Ilg, and S. Hess, J. Phys.: Condens Matter 15, S1403 (2003).

[4] M.A. Martsenyuk, Yu.L. Raikher, and M.I. Shliomis, Zh. Eksp. Teor. Fiz. 65, 834 (1973) [Sov. Phys. JETP 38, 413 (1974)].

[5] A.Yu. Zubarev and L.Yu. Iskakova, JETP 80, 857 (1995).

[6] A.Yu. Zubarev and L.Yu. Iskakova, Phys. Rev. E 61, 5415 (2000).

[7] A.Yu. Zubarev, JETP 93, 80 (2001).
[8] S. Odenbach, J. Fleischer, and A. Yu. Zubarev, Phys. Rev. E (to be published).

[9] M. Doi and H. See, J. Phys. Soc. Jpn. 61, 2090 (1992).

[10] H. See, J. Phys. D 33, 1414 (2000).

[11] P. Ilg and M. Kröger, Phys. Rev. E 66, 021501 (2002).

[12] J.T. Waldron, Yu.P. Kalmykov, and W.T. Coffey, Phys. Rev. E 49, 3976 (1994).

[13] P. Ilg, M. Kröger, and S. Hess, J. Chem. Phys. 116, 9078 (2002).

[14] P. Ilg, M. Kröger, and S. Hess, Magnetohydrodynamics 39, 41 (2003).

[15] A. Cebers, Magnetohydrodynamics (N.Y.) 20, 349 (1984). 